\title{
Neural Correlates of Antidepressant-Related Sexual Dysfunction: A Placebo-Controlled fMRI Study on Healthy Males Under Subchronic Paroxetine and Bupropion
}

\author{
Birgit Abler*, 1,4, Angela Seeringer ${ }^{2,4}$, Antonie Hartmann', Georg Grön', Coraline Metzger ${ }^{3}$, Martin Walter ${ }^{3}$ \\ and Julia Stingl ${ }^{2}$
}

'Department of Psychiatry, University of Ulm, Ulm, Germany; ${ }^{2}$ Institute of Pharmacology of Natural Products and Clinical Pharmacology,

University of Ulm, Ulm, Germany; ${ }^{3}$ Department of Psychiatry, Otto von Guericke-University, Magdeburg, Germany

\begin{abstract}
Sexual dysfunction is a common side effect of selective serotonin reuptake inhibitors (SSRIs) like paroxetine in the treatment of depression, imposing a considerable risk on medication adherence and hence therapeutic success. Bupropion, a norepinephrine and dopamine reuptake inhibitor, is recommended as an alternative treatment without adverse effects concerning sexual arousal and libido. We investigated the neural bases of paroxetine-related subjective sexual dysfunction when compared with bupropion and placebo. We scanned 18 healthy, heterosexual males in a randomized, double-blind, within-subject design while watching video clips of erotic and nonerotic content under steady-state conditions after taking $20 \mathrm{mg}$ of paroxetine, $150 \mathrm{mg}$ of bupropion, and placebo for 7 days each. Under paroxetine, ratings of subjective sexual dysfunction increased compared with placebo or bupropion. Activation along the anterior cingulate cortex (ACC), including subgenual, pregenual, and midcingulate cortices, in the ventral striatum and midbrain was decreased when compared with placebo. In contrast, bupropion let subjective ratings and ACC activations unchanged and increased activity of brain regions including posterior midcingulate cortex, mediodorsal thalamus, and extended amygdala relative to placebo and paroxetine. Brain regions that have been related to the processing of motivational (ventral striatum), emotional, and autonomic components of erotic stimulation (anterior cingulate) in previous studies showed reduced responsiveness under paroxetine in our study. Drug effects on these regions may be part of the mechanism underlying SSRI-related sexual dysfunction. Increased activation under bupropion may point to an opposite effect that may relate to the lack of impaired sexual functioning.

Neuropsychopharmacology (20II) 36, 1837-1847; doi: I0.1038/npp.20II.66; published online 4 May 201 I
\end{abstract}

Keywords: bupropion; paroxetine; erotic stimulation; sexual dysfunction; $\mathrm{fMRI}$

\section{INTRODUCTION}

Selective serotonin reuptake inhibitors (SSRIs) are the most widely prescribed antidepressant drugs (Bauer et al, 2008). For the majority of relevant diagnoses, regular intake is recommended for months or even longer (Gaebel and Falkai, 2001). However, despite the positive profile of SSRIs, premature discontinuation is common and frequently discussed to account for nonresponse and relapse (Bull et al, 2002). Sexual dysfunction is a typical side effect of SSRI treatment (Serretti and Chiesa, 2009) that conditions discontinuation and may represent the most clinically

*Correspondence: Dr B Abler, Department of Psychiatry, University of Ulm, Leimgrubenweg 12-|4, 89075 Ulm, Germany, Tel: + 49 (0) 731 500 61560, Fax: + 49 (0) 731 500 61412,

E-mail: birgit.abler@uni-ulm.de

${ }^{4}$ These authors contributed equally to this work.

Received 25 October 2010; revised 28 February 2011; accepted 22 March 201। relevant side effect among patients in their twenties or thirties (Bishop et al, 2006). Decreased sexual desire and interest in sexual activity along with erectile dysfunction is reported in up to $60 \%$ of treatments with SSRIs (Gregorian et al, 2002) with the highest rates of up to $64 \%$ observed under paroxetine (Clayton et al, 2002). As sexual dysfunction is similarly reported under treatment with dual antidepressants inhibiting serotonin and norepinephrine reuptake (Montejo et al, 2001), but not under selective norepinephrine reuptake inhibitors (Clayton et al, 2003), the serotonergic component seems to represent the crucial part in mediating sexual dysfunction.

Bupropion is a selective dopamine and norepinephrine reuptake inhibitor and is named as an alternative that is reportedly not associated with side effects on sexual functioning (Coleman et al, 2001). Bupropion has even been suggested as a potential treatment for SSRI-induced sexual dysfunction or hypoactive sexual desire disorder in women (Modell et al, 2000). This is in line with clinical 
observations relating dopamine agonists to hypersexuality (Voon et al, 2009) and dopamine antagonists to sexual dysfunction (Baldwin and Mayers, 2003). Animal experiments clearly support the notion that modulation of central, but not peripheral, serotonergic pathways is involved in SSRI-related alteration of sexual functioning (Waldinger et al, 1998). Enhancement of central serotonergic neurotransmission with stronger activation of inhibitory postsynaptic autoreceptors (Blier et al, 1988) has been postulated as a likely SSRI effect modulating the inhibitory control of ejaculation (Giuliano and Clement, 2006). However, the actual pathophysiology of SSRI-related sexual dysfunction is poorly understood. Using functional magnetic resonance imaging (fMRI), we investigated the neural correlates of erotic stimulation under paroxetine. Because sexual dysfunction, including decreased libido and anorgasmia, is a common symptom of depression irrespective of antidepressant treatment, we investigated healthy subjects under subchronic treatment. To control for the effects of serotonergic $v s$ dopaminergic/noradrenergic antidepressant treatment, we set up a randomized crossover design with each subject receiving paroxetine, bupropion and placebo over the course of 1 week separated by sufficient washout phases. Stimulation with erotic video clips has already been demonstrated as a reliable tool to investigate brain regions related to sexual functioning (Redoute et al, 2000) and to contrast medication effects on these brain regions in patients suffering from depression (Kim et al, 2009). Under placebo, we expected to find activation in those brain regions that are reported to be active during erotic stimulation according to previous fMRI studies, that is, the anterior cingulate cortex (ACC), lateral occipital cortex, insula, amygdala, hypothalamus, thalamus, and the ventral striatum (Arnow et al, 2002; Ferretti et al, 2005; Moulier et al, 2006; Mouras, 2004; Redoute et al, 2000; Walter et al, $2008 \mathrm{a}, \mathrm{b})$. In line with previous studies of blunted ventral striatal activation under SSRIs (McCabe et al, 2010), paroxetine was expected to exert inhibitory effects on reward system activation, the latter being identified as specifically elicited by erotic stimulation as a primary reward (Walter et al, 2008a). In contrast, bupropion, as a dopamine reuptake inhibitor, was expected to enhance activation of the dopaminergic reward system as reported for the administration of L-dopa or dopamine agonists in healthy subjects (Pessiglione et al, 2006). SSRI-related attenuation of ventral ACC and insula was expected from recent findings of similar effects of subchronic administration of citalopram on emotional anticipation in healthy subjects (Simmons et al, 2009).

\section{SUBJECTS AND METHODS}

\section{Subjects}

A total of 23 healthy male, heterosexual subjects (3 lefthanded) were randomized to take placebo, bupropion, and paroxetine at routine clinical doses for 7 days each in a counterbalanced order. After each medication period, these subjects were scheduled to take part in the fMRI experiment. From this sample, 18 subjects aged 23-34 years (mean 25.4, SD 2.9, 3 left-handed) completed all three fMRI sessions (see Figure 1) and were therefore included in the final analyses. Two of the five subjects who did not enter the final analyses were excluded because data from one of the three fMRI session could not be considered due to technical problems (spiking, coil malfunction). Two further subjects were excluded from the analyses because blood levels of paroxetine were too low to be quantified rendering intake of the drug doubtful. One subject had to be excluded because the blood sample for the bupropion levels was lost. The mean plasma bupropion level of included subjects was $59.9 \mathrm{ng} / \mathrm{ml}$ (SD 27.7) and the mean plasma paroxetine level was $28.1 \mathrm{ng} / \mathrm{ml}$ (SD 17.9). Blood samples were taken after each fMRI session, which is $\sim 3 \mathrm{~h}$ after drug intake. Plasma levels were assessed as an estimate of drug exposure and drug adherence. More precise pharmacological profiles would have meant taking more blood samples that, however, was not compatible with the present study design. Blood levels were not analyzed before completion of the whole study.

The study was approved by the local ethical review board of the University of Ulm. All volunteers gave written informed consent before the study in accordance with the Declaration of Helsinki Principles. Each participant received a full medical evaluation including a medical history, a physical examination, and a Structured Clinical Interview for DSM-IV Axis I Psychiatric Disorders (SCID), as well as laboratory blood tests and an electrocardiogram to exclude cardiac, renal, or hepatic preconditions. Exclusion criteria were any current or past psychiatric or neurological illnesses, any serious general medical condition, use of illegal drugs, and excessive consumption of caffeine or alcohol ( $>14$ units/week). Subjects were also excluded for any significant baseline sexual dysfunction or sexual disorders (see Supplementary Materials for details). One subject took a single dose of acetaminophen, one a single dose of metamizole, and one used a steroid inhaler for mild allergic asthma parallel to the study medication. Other than that, none of the subjects took any medication and none had taken any other medication within the week before the study. Subjects were asked to refrain from alcohol parallel to the study medication and from coffee on the day of the scans. Upon recruitment, subjects completed two self-report scales: the Center for Epidemiologic Studies Depression Scale (CES-D; Radloff, 1977), with the German version (Allgemeine Depressions Skala/ADS; Hautzinger and Bailer, 1993), and a German version (Reinecke et al, 2006) of the Massachusetts General Hospital Sexual Function Questionnaire (MGH-SFQ; Labbate and Lare, 2001). The MGH-SFQ is based on self-report and assesses sexual interest, sexual arousal, ability to achieve orgasm, ability to achieve and maintain an erection, and overall sexual satisfaction over the past month. The questionnaire was modified to reflect subjective sexual dysfunction only over the past week of medication.

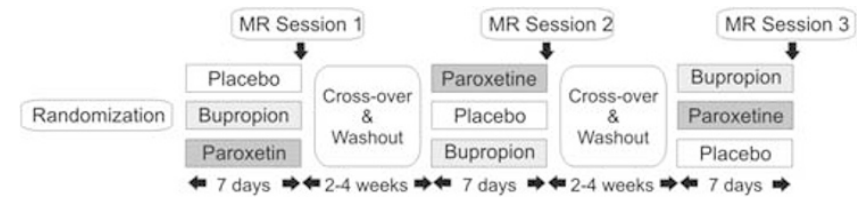

Figure I Study design. To each of the three randomization arms, six subjects were assigned. 


\section{Study Design and Procedures}

The entire study was performed as a randomized, doubleblind, placebo-controlled, within-subject, crossover design (Figure 1). Each subject was investigated on three different occasions, once under placebo (PLAC: gelatine capsule filled with mannitol powder), once under paroxetine (PAR: $20 \mathrm{mg}$, identical capsule with pulverized paroxetine), and once under bupropion (BUP: $150 \mathrm{mg}$, identical capsule with bupropion) at intervals of at least 14-day washout time. For each treatment, subjects were instructed to take one capsule each morning for 7 days to reach steady-state blood levels. SSRI-related changes of sexual functioning have been reported after single doses concerning premature ejaculation (Patel and Hellstrom, 2009) as well as after 8 days of intake concerning a broader spectrum of sexual functions including libido and sexual arousal (Dunn et al, 2007). On the seventh day, subjects were scheduled for an fMRI scan $2 \mathrm{~h}$ after intake of the last capsule.

\section{Task and Stimuli}

In the scanner, a standard block design with prolonged visual stimulation using erotic and neutral video clips was used. Erotic video clips showed sexual interactions between one man and one or two women (petting, oral sex, and vaginal intercourse) extracted from commercial adult films. The neutral video clips depicted men and women in emotionally neutral, nonerotic interactions (at a shop, at the airport). Nine video clips of each type were presented at a length of $20 \mathrm{~s}$ each, separated by a 20-s interstimulus interval with a white fixation cross shown on a black screen. Video clips were presented in a pseudo-randomized order with a maximum of two consecutive clips of the same type.

\section{fMRI Acquisition}

A 3.0 Tesla Magnetom ALLEGRA Scanner (Siemens, Erlangen, Germany) equipped with a head coil was used to acquire $\mathrm{T} 1$ anatomical volume images $(1 \times 1 \times 1 \mathrm{~mm}$ voxels) and functional magnetic resonance images. Twenty three transversal slices were acquired with an image size of $64 \times 64$ pixels and a field of view of $192 \mathrm{~mm}$. Slice thickness was $3 \mathrm{~mm}$ with $0.75 \mathrm{~mm}$ gap resulting in a voxel size of $3 \times 3 \times 3.75 \mathrm{~mm}$. Images were centered on basal structures of the brain including subcortical regions of interest (basal ganglia and prefrontal regions). Functional images were recorded using $\mathrm{T} 2{ }^{*}$-sensitive gradient echo echo-planar imaging measuring changes in BOLD contrast. In all, 487 volumes were obtained during viewing of video clips at a TR of $1500 \mathrm{~ms}$ (TE $35 \mathrm{~ms}$, flip angle $90^{\circ}$ ).

\section{fMRI Analysis}

Image preprocessing and statistical analyses were carried out using Statistical Parametric Mapping (SPM5, Wellcome Trust Centre for Neuroimaging, London, UK) with a random effects model for group analyses. Preprocessing of the individual functional scans included realignment to correct for motion artifacts, slice timing, spatial normalization to a standard template (Montreal Neurological Institute, MNI), and smoothing with an $8 \mathrm{~mm}$ full width at half maximum (FWHM) Gaussian kernel. Intrinsic autocorrelations were accounted for by $\mathrm{AR}(1)$ and lowfrequency drifts were removed via high-pass filtering.

After preprocessing, first-level analyses were performed for each subject. According to the general linear model we defined regressors to analyze each of the two types of video stimuli (erotic and nonerotic). Video blocks were modeled as timely extended events of $20 \mathrm{~s}$ and convolved with the hemodynamic response function. The six realignment parameters modeling residual motion were also included in the individual models. The individual contrast images for the erotic and nonerotic condition were then included in a second-level group analysis using an ANOVA model with condition (EROTIC/NONEROTIC) as the first factor. Treatment (PLAC/BUP/PAR) was added as a second factor with three levels to test on significant interaction effects of medication on the contrast of erotic $v s$ nonerotic stimuli. Inference of significant treatment effects was computed using directed condition-by-treatment $t$-contrasts for pairwise differences between treatment levels. To account for the post hoc character of these tests, their computation was inclusively masked by an omnibus F-test on conditionby-treatment interactions within the $2 \times 3$ ANOVA. The statistical threshold for this mask was set to a value of $p<0.005$ uncorrected to avoid false-negative results due to overly strict thresholding. However, significant treatment effects were inferred only if results of the post hoc $t$-contrasts survived a statistical threshold of $p<0.05$ false discovery rate (FDR) corrected to account for multiple comparisons, and if clusters of significant voxels comprised at least 10 contiguously significant voxels. In a second step, a conservative omnibus F-test was applied thresholded at $p<0.05$ with FDR corrections for multiple comparisons.

\section{Neuropsychological Testing and Additional Questionnaires}

After scanning and blood sampling, subjects performed three simple reaction time tasks (subtests from the Test Battery for Attentional Performance, Zimmermann \& Fimm, Psychologische Testsysteme, Herzogenrath, Germany): simple reaction to a visual stimulus (RT1), reaction to a visual stimulus after a warning tone (RT2), and divided attention (RT3), involving reaction to distinct visual and auditory stimuli at the same time. Reaction time tasks were applied to assess for general medication effects on attention and concentration as potential moderators of treatment effects. Subjects also completed the self-report questionnaires on side effects and sexual functioning (MGH-SFQ, for detailed description see supplements) during the past week on each occasion. Cumulative ratings on the MGH-SFQ ranged from 5 (minimal value: improvement of sexual functioning) over 10 (sexual functioning unchanged compared with normal) to 30 (maximal value: sexual functioning markedly impaired compared with normal). Ratings above 10 indicate subjectively impaired sexual functioning when compared with normal in the sense of subjective sexual dysfunction. Subjective side effects were additionally assessed in a medical interview with open questions and a structured part (UKU side-effects scale; Lingjaerde et al, 1987) by one of the study physicians (BA or AS) on each visit. Repeatedmeasures ANOVAs and post hoc paired $t$-tests were applied to analyze reaction time and questionnaire results. 


\section{RESULTS}

\section{Questionnaires and Neuropsychological Tasks}

Results from the ADS scale (mean: 6.9, SD 5.0) confirmed that none of the subjects suffered from depression at the time of the study.

Of the subjects, $56 \%$ complained about side effects of any kind under placebo according to the UKU side-effects scale. There were complaints over a wide range of different symptoms at a rate of $67 \%$ under bupropion and of $83 \%$ under paroxetine, without reaching significance when compared with placebo (ANOVA: $\mathrm{F}(2,34)=2.58, p=0.09$ ). Particularly, subjective ratings of sedation did not differ (one-way ANOVA: $\mathrm{F}(2,34)=2.06, p=0.14$ ) between treatments.

Upon enrollment, subjectively rated sexual function as assessed by the mean cumulative score of the MGH-SFQ was 11.06 (SD 1.7). Above normal values (one-sample $t$-test, $p<0.05$ ) of more than 2 points per subscale were observed only for question (5) concerning sexual satisfaction $($ mean $=2.67, \mathrm{SD}=1.0)$, indicating minimal sexual dissatisfaction. However, subjective MGH-SFQ ratings differed significantly between medications (repeated measurements ANOVA: $\mathrm{F}(2,34)=5.05, p=0.01)$, with average overall scores of 12.0 (SD 2.4) under placebo, 11.8 (SD 2.9) under bupropion, and 14.6 (SD 4.3) under paroxetine (Figure 2a). Post hoc paired $t$-tests confirmed significantly higher overall scores under paroxetine when compared with placebo $(\mathrm{t}(17)=2.58, p=0.02)$ or bupropion $(\mathrm{t}(17)=2.30$, $p=0.03$ ). No significant differences were observed when bupropion was compared against placebo. Analyzing rating scores of each of the five MGH-SFQ subscales revealed a significant interaction effect of factors treatment and scale (repeated measurements ANOVA: $\mathrm{F}(8,136)=2.41, p=0.02$ ) with increases of subjective ratings (paired $t$-tests, $p<0.05$ ) concerning diminished sexual arousal and diminished ability to achieve orgasm under paroxetine when compared with both enrollment and placebo ratings (Figure $2 b$ ). It is of note that the ability to get an erection was not affected by any of the treatments. Furthermore, none of the symptoms caused marked distress or interpersonal difficulty or otherwise justified a clinical diagnosis of sexual dysfunction.

Mean reaction times in the neuropsychological tests after scanning did not differ between treatments for the tests RT1 and RT2 (simple reaction without/with warning tone, oneway ANOVAs: RT1: $\quad \mathrm{F}(2,34)=0.11, \quad p=0.89 ; \quad \mathrm{RT} 2$ : $\mathrm{F}(2,34)=0.65, p=0.53)$. For RT3, divided attention, we found a significant effect of medication $(\mathrm{F}(2,34)=4.40$, $p=0.02)$ ). Mean reaction times under placebo (mean $601.7 \mathrm{~ms}$, SD 56.7) were significantly faster than under both drugs, bupropion (mean $629.3 \mathrm{~ms}$, SD $52.0 ; \mathrm{t}(17)=2.46$, $p=0.02$ ) and paroxetine (mean $642.7 \mathrm{~ms}$, SD 69.8, $\mathrm{t}(17)=3.11, p=0.006)$. No significant difference was found for bupropion $v s$ paroxetine $(\mathrm{t}(17)=0.78, p=0.45)$.

\section{fMRI Results}

Main effect of erotic stimulation under placebo. Contrasting erotic minus nonerotic visual stimulation under placebo revealed significantly $(p<0.05$ FDR corrected) increased activations along the ACC in the amygdala, hypothalamus, sexual dysfunction

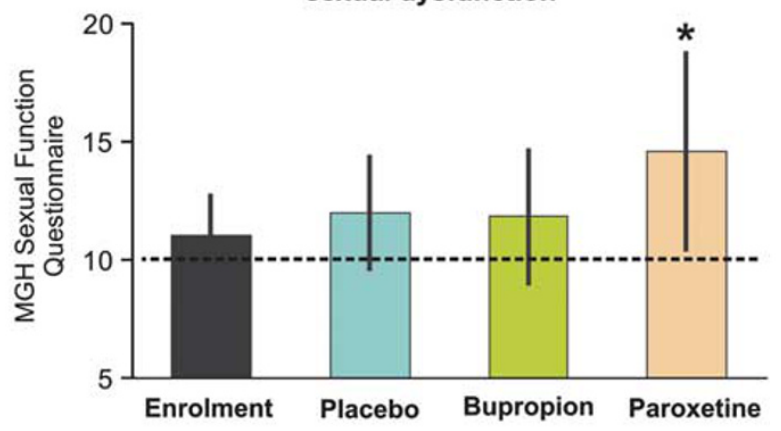

b Subscales ratings of subjective sexual dysfunction

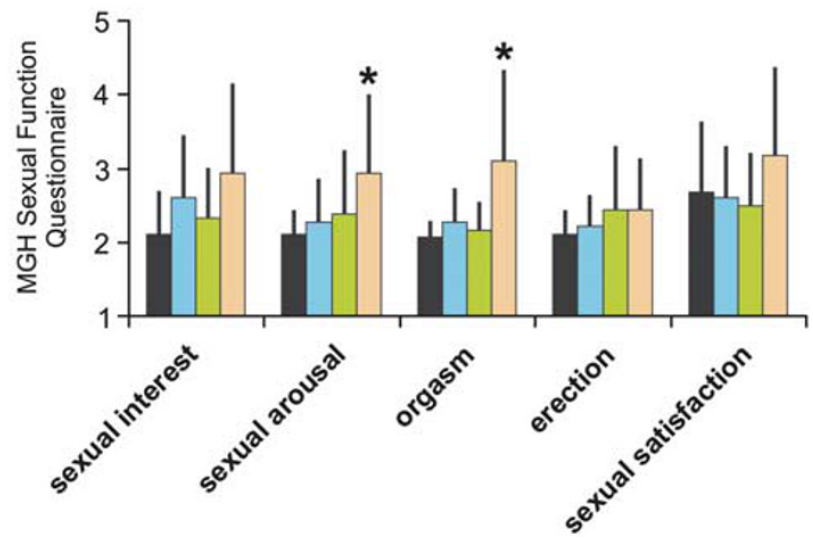

Figure 2 (a) Group-averaged cumulative rating scores obtained from the five-item Massachusetts General Hospital Sexual Function Questionnaire (MGH SFQ). Overall, subjective sexual dysfunction was significantly $\left({ }^{*} p<0.05\right)$ increased under paroxetine during the past week. A minimal cumulative score of 5 indicates increased sexual interest when compared with normal; 'average' cumulative score of 10 indicates no change in sexual functioning compared with before (dashed black line); scores above 10 indicate decreased sexual functioning; the maximal cumulative score of 30 has the meaning of no sexual interest. (b) Group-averaged ratings for each subscale. MGH-SFQ subscales ratings indicate diminished $(* p<0.05)$ ability to get sexually aroused and to achieve orgasm under paroxetine when compared with enrollment, placebo (arousal and orgasm), and bupropion (orgasm only).

ventral striatum, midbrain, lateral occipital cortex, precuneus, and the fusiform gyrus.

Treatment effects. Significant results $(p<0.05$, FDR corrected) of the directed post hoc tests on pairwise differences between treatment levels are summarized in Table 1. Compared with placebo, decreased activation under paroxetine was significant in midbrain structures, the ventral striatum, as well as in three different sections along the cingulate gyrus (sgACC, pgACC, and aMCC). Comparing bupropion against placebo, only aMCC activation was decreased whereas the pMCC showed increased activity. Contrasting bupropion against paroxetine, increased activation was evident in the sub- and pre-genual ACC as well as in the pMCC. Differential signals along the cingulate gyrus under the three different treatments are depicted in Figure 3. Furthermore, bupropion was associated with increased activation compared with both paroxetine and placebo in various brain regions including the SLEA, 
Table I Significant Condition-by-Treatment Interaction Effects

\begin{tabular}{|c|c|c|c|c|c|c|c|c|c|c|c|c|}
\hline \multirow{2}{*}{ Video: erotic vs nonerotic stimuli } & \multicolumn{3}{|c|}{ Placebo $>$ paroxetine } & \multicolumn{3}{|c|}{ Placebo > bupropion } & \multicolumn{3}{|c|}{ Bupropion $>$ placebo } & \multicolumn{3}{|c|}{ Bupropion $>$ paroxetine } \\
\hline & $z$ & NV & $\begin{array}{c}\text { Peak Coord. } \\
x / y / z\end{array}$ & $z$ & NV & $\begin{array}{c}\text { Peak Coord. } \\
x / y / z\end{array}$ & $z$ & NV & $\begin{array}{c}\text { Peak Coord. } \\
x / y / z\end{array}$ & $z$ & NV & $\begin{array}{c}\text { Peak Coord. } \\
\qquad x / y / z\end{array}$ \\
\hline \multicolumn{13}{|l|}{ Cingulate cortex } \\
\hline Subgenual ACC* & 4.21 & 46 & $-12 / 32 /-6$ & & & & & & & 3.61 & 37 & $-14 / 32 /-6$ \\
\hline Posterior MCC** & & & & & & & 3.30 & 102 & $16 / 20 / 34$ & 4.43 & 119 & $16 / 24 / 36$ \\
\hline \multicolumn{13}{|l|}{ Subcortical structures } \\
\hline Midbrain* & 4.72 & 150 & $-4 /-24 /-14$ & & & & & & & 5.29 & 485 & $-4 /-24 /-14$ \\
\hline Ventral striatum, right & 3.04 & 16 & $6 / 14 /-6$ & & & & & & & 3.66 & 30 & $10 / 12 /-6$ \\
\hline Ventrolateral thalamus, left* & & & & & & & 3.80 & 285 & $-22 /-14 / 14$ & 4.33 & 299 & $-22 /-14 / 14$ \\
\hline Dorsomedian thalamus & & & & & & & 2.43 & a & $-6 /-12 / 8$ & 3.74 & a & $-4 /-10 / 6$ \\
\hline \multicolumn{13}{|l|}{ Cortical activations } \\
\hline Lateral orbital FC* (BAI I/47) & & & & & & & 3.64 & 58 & $-36 / 40 /-8$ & 4.04 & 61 & $-34 / 36 /-6$ \\
\hline Medial FC, BA 6 & & & & & & & 3.70 & 140 & $52 / 0 / 30$ & 3.60 & 129 & $56 / 6 / 34$ \\
\hline Medial/superior temporal gyrus* (BA 39/40) & & & & & & & 3.79 & 268 & $38 /-56 / 30$ & 4.55 & 271 & $36 /-58 / 28$ \\
\hline Fusiform gyrus, left (BA 19/37) & & & & & & & 3.50 & 44 & $-34 /-62 /-4$ & 3.79 & 44 & $-36 /-62 /-6$ \\
\hline Fusiform gyrus, right* (BA 19/37) & & & & & & & & & & 3.76 & 56 & $38 /-40 /-12$ \\
\hline Parahippocampus, left* & & & & & & & 3.58 & 450 & $-22 /-34 /-6$ & 4.38 & 450 & $-20 /-34 /-6$ \\
\hline Parahippocampus, right & & & & & & & 4.00 & 142 & $42 /-20 /-14$ & 2.85 & 61 & $40 /-22 /-10$ \\
\hline
\end{tabular}

Z: z-score; NV: number of contiguously significant voxels; peak coordinates of clusters are MNI (Montreal Neurological Institute) normalized stereotactic coordinates; $-x$ : left from the anterior commissure (AC); -y: posterior from AC; $-z$ : inferior from AC; ACC: anterior cingulate cortex; MCC: midcingulate cortex; FC: frontal cortex; BA: Brodman area.

The table shows results of post hoc testing of pairwise differences between treatments at $p<0.05$, FDR corrected. The post hoc testing was locally constrained to voxels surviving a threshold of $p<0.005$ in the $2 \times 3$ ANOVA with factors condition (erotic and nonerotic) and treatment. Brain areas with significant interaction effects in the $2 \times 3$ ANOVA even at the more conservative threshold of $p<0.05$, FDR corrected, are marked with $(*)$. No significant interactions were observed for paroxetine $>$ placebo or paroxetine $>$ bupropion.

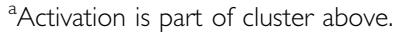

ventrolateral and dorsomedian thalamus, parahippocampus, lateral orbitofrontal cortex, and fusiform gyrus (Table 1).

To test on the reliability of the inclusive mask $(p<0.005$, uncorrected) used for the post hoc testing above, the same omnibus F-test on treatment effects used to create the mask was replicated at a statistical threshold of $p<0.05$, FDR corrected. This test revealed again significant effects in the midbrain, the sub- and pre-genual ACC (sgACC and pgACC), the anterior and posterior midcingulate cortex (aMCC and pMCC), the left sublenticular extended amygdala (SLEA), the left ventral striatum, and the left parahippocampal area (see also Table 1).

To further investigate drug effects on the fMRI signal, signal time courses were extracted from three prototypical regions (Figure 4): (1) the midbrain that, like rostral ACC regions, showed decreased activations under paroxetine when compared with placebo and bupropion, (2) the extended amygdala as a representative brain region for the network with increased activation under bupropion, and (3) the aMCC as the only brain region with decreased activation under both drugs compared with placebo. Paired $t$-tests of differences in signal time courses at single time points indicated prolonged differential effects in midbrain and particularly the extended amygdala under bupropion when compared with placebo. Differential effects in all three brain regions were most pronounced in the first part of the video stimulation.

Correlation analyses. Whole-brain analyses did not reveal any significant correlations between functional activations and subjective ratings, reaction time data, or medication 

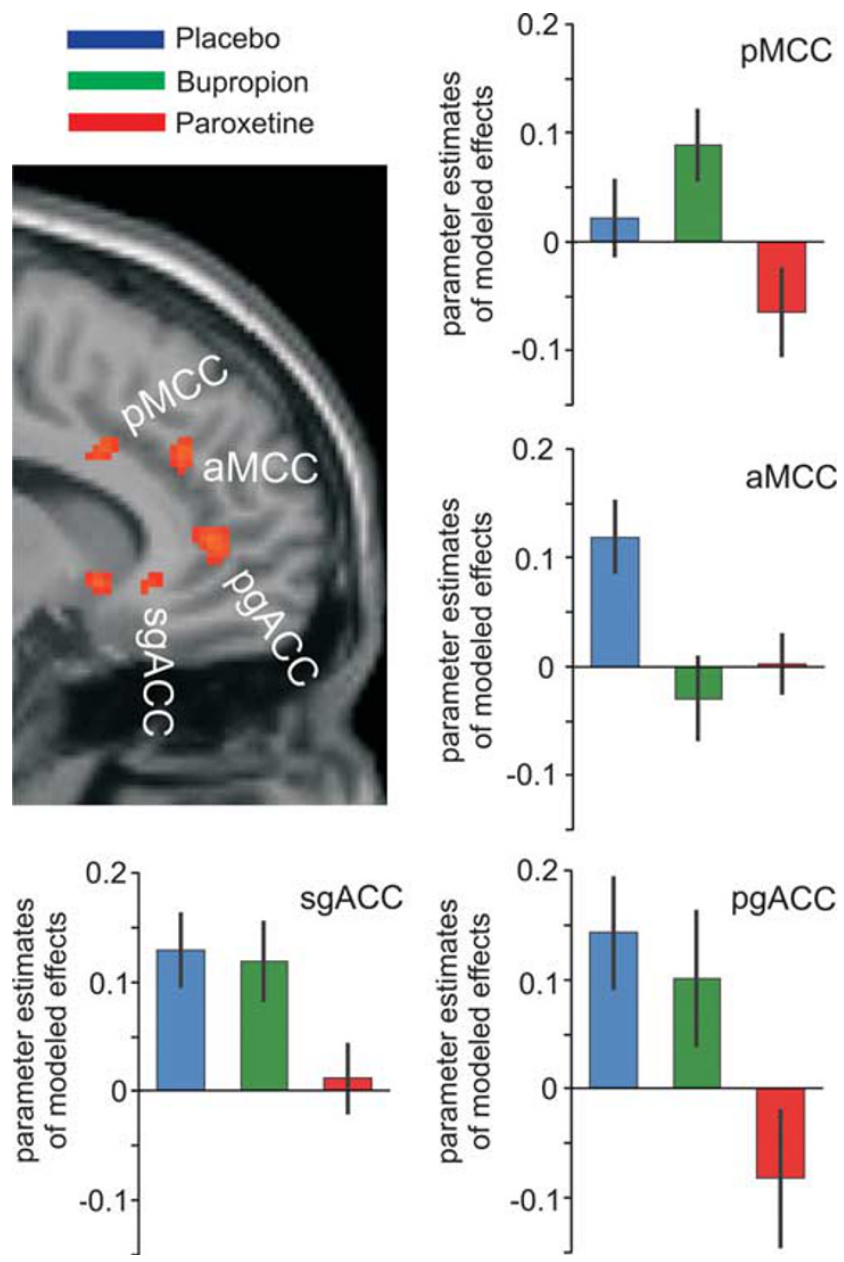

Figure 3 Significant treatment effects ( $p<0.05$ FDR corrected) along the anterior cingulate gyrus as revealed by an appropriate omnibus F-test on treatment effects for placebo, bupropion, and paroxetine (for demonstration purposes, the threshold was lowered to $p<0.005$ uncorrected). Parameter estimates of modeled effects were extracted from the peak voxel of each cluster, that is, the subgenual anterior cingulate cortex (sgACC), the pregenual anterior cingulate cortex (pgACC), the anterior midcingulate cortex (aMCC), and the posterior midcingulate cortex (pMCC). Activation decreased under paroxetine when compared with placebo and bupropion in sgACC, pgACC, and pMCC. In aMCC, activation decreased under both drugs compared with placebo.

blood levels (even when tested at different, rather liberal thresholds). However, a significant correlation (Figure 5) was observed between total MGH-SFQ score and averaged parameter estimates obtained from the pMCC region (voxels significant in the omnibus F-test at $p<0.05$ corrected). This region had been selected for further investigation based on previous literature showing correlations between brain activation in the MCC with behavioral measures of sexual functioning (Redoute et al, 2000; Moulier et al, 2006; Georgiadis et al, 2010). The more the brain activation decreased under paroxetine compared with placebo, the more overall ratings of sexual dysfunction (paroxetine minus placebo) increased $(r=0.42 ; p=0.041)$. Furthermore, individual blood levels of paroxetine were also significantly $(r=0.40, p=0.050)$ correlated with the differential neural activation (Figure 5). No further significant correlations with rating scores, blood levels, or reaction times were observed in other cingulate areas significant in the above interaction analysis (particularly, not the aMCC). Computation of meaningful correlations between brain activations and behavioral ratings under bupropion was not possible because of the small variance of the rating scores with zero differences of ratings (placebo minus bupropion) in half of the subjects.

\section{DISCUSSION}

We investigated the effects of 1-week administration of the SSRI paroxetine and the dopamine and norepinephrine reuptake inhibitor bupropion in a double-blind, placebocontrolled, crossover design in healthy male volunteers. We observed increased subjective ratings of sexual dysfunction under paroxetine compared with placebo and bupropion. Specifically, subjective sexual arousal and the ability to achieve orgasm were significantly impaired under paroxetine whereas the ability to get an erection, and ratings of sexual interest and sexual satisfaction were not significantly altered under this drug.

Behavioral effects were accompanied by specific functional alterations in brain regions previously associated with sexual function in humans. Brain activation related to dynamic visual erotic stimulation under medication revealed antipodal results in two different networks. Compared against placebo and bupropion, paroxetine attenuated brain activation in neural structures comprising the midbrain, the nucleus accumbens, and sub- and pre-genual ACC. In contrast, bupropion led to an enhanced and prolonged activation in the parahippocampus, the amygdala (SLEA), thalamus, and fusiform gyrus when compared against placebo or paroxetine. Bupropion and paroxetine were associated with reduced activation within the anterior MCC, and activation of the posterior MCC upon erotic stimulation was increased under bupropion. Individual parameters of decreased neural activation in the pMCC under paroxetine correlated with individually increased overall ratings of sexual dysfunction and with individual drug concentrations obtained from plasma samples.

\section{Blunted Anterior Cingulate Activation Under Paroxetine}

According to the four-region neurobiological model of human cingulate cortex architecture (Palomero-Gallagher et al, 2009; Vogt et al, 2003), the four cingulate foci with differential medication effects are related to areas along the cingulate gyrus with distinct cytoarchitectonic and functional properties. This model postulates that sgACC and pgACC are reciprocally connected with the amygdala and are involved in emotional and autonomic processing. The aMCC is associated with reward processing and is active during fear. The pMCC has been related to error detection and is not primarily activated by simple emotions (Phan et al, 2002; Vogt et al, 2003). Paroxetine led to decreased fMRI activations in all of these regions along the cingulate gyrus. Particularly sgACC, with its high density of 5-HT1A receptors in Brodman area 25 being the primary target of SSRI treatment (Haddjeri et al, 1998) has been postulated to be critical for understanding the effects of antidepressant 

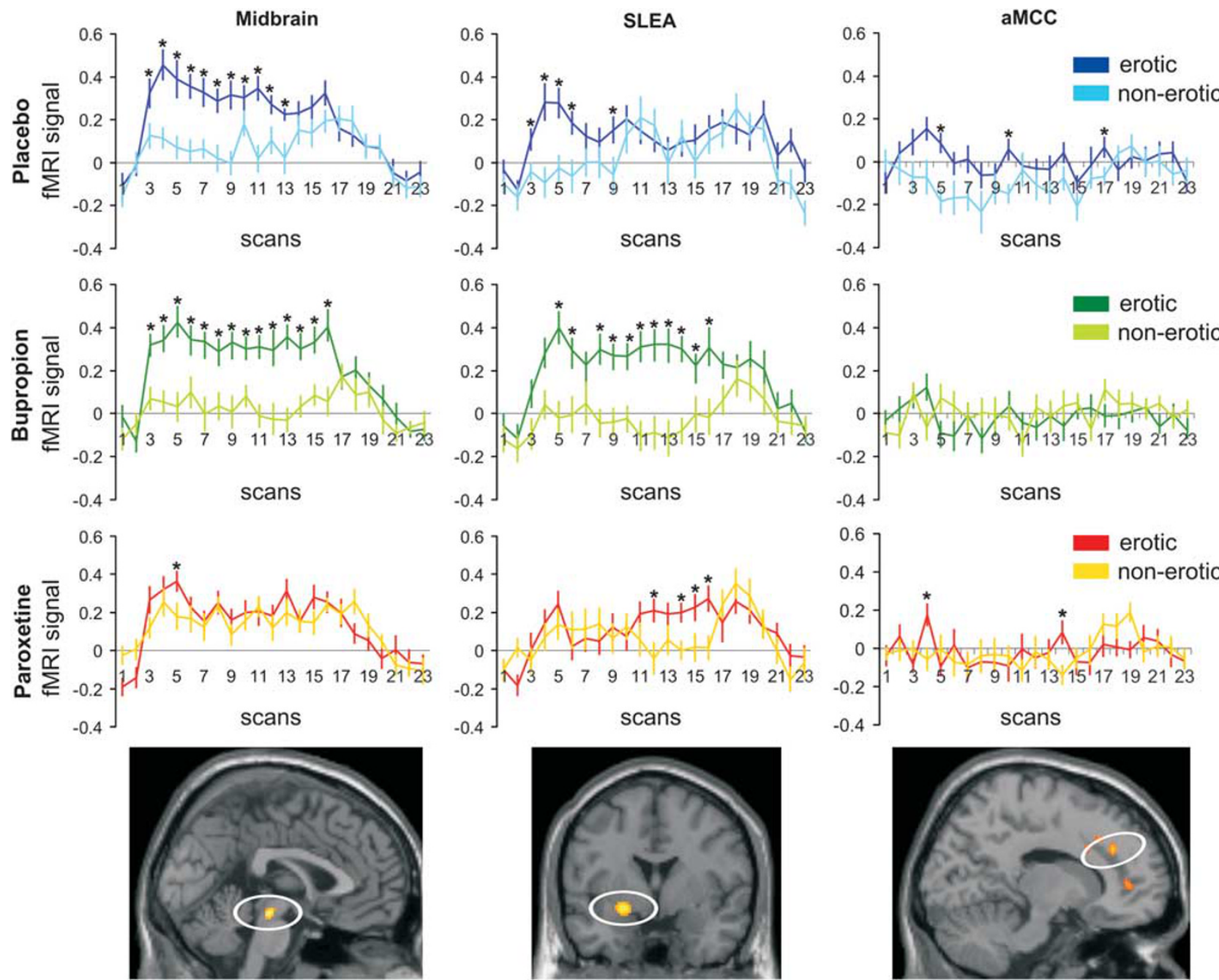

Figure 4 Statistical parametric maps (SPMs) and FMRI signal time courses extracted from three prototypical regions significantly associated with erotic visual stimulation with differential drug effects in an omnibus F-test $(p<0.05$ FDR corrected): midbrain with decreased activation under paroxetine, sublenticular extended amygdala (SLEA) with increased and prolonged activation under bupropion, and decreased activation under paroxetine; anterior midcingulate cortex (aMCC) with decreased activation under both drugs compared with placebo. The depicted SPMs are thresholded at $p<0.005$ uncorrected for demonstration purposes. Mean first eigenvariate values were extracted from the clusters for each subject and then averaged. *Significant difference at $p<0.05$ in a paired $t$-test of the $\mathrm{fMRI}$ signal at each time point.
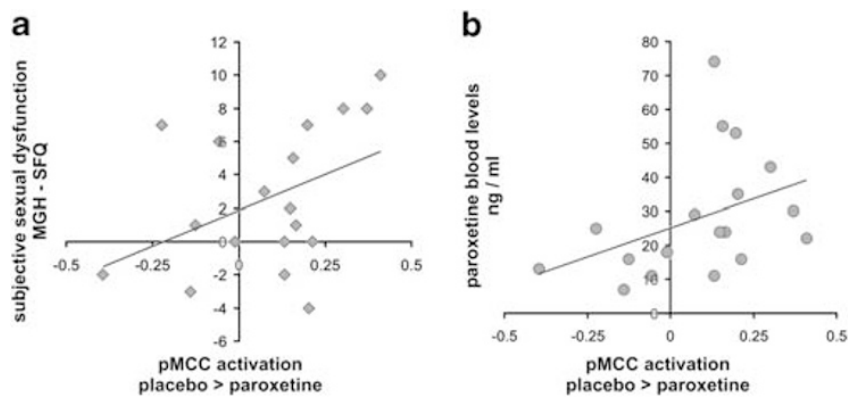

Figure 5 Significant $(p<0.05)$ correlations of $\mathrm{fMRI}$ activation in the PMCC region of interest (parameter estimates: placebo > paroxetine) with (a) increases in ratings of subjective sexual dysfunction under paroxetine relative to placebo and (b) with paroxetine blood levels.

therapy (Ressler and Mayberg, 2007). Decreased sgACC activation under paroxetine is in line with findings of treatment effects in depression (Mayberg et al, 2005), and with findings of another fMRI study investigating the effects of subchronic SSRI administration in healthy subjects using emotional faces (Arce et al, 2008). This may point to a receptor-specific but functionally more general SSRI effect independent of the type of stimulus material.

Like the sgACC, the more rostral pgACC has also been found to be modulated by subchronic escitalopram administration upon aversive stimuli (Simmons et al, 2009). Activity of the pgACC has also been postulated to correlate with subjective sexual intensity and its hedonic value (Walter et al, 2008a) and was identified as a brain region specifically coding the interaction of sexual intensity and emotional value of a stimulus. In Redoute's model of sexual arousal, activation of rostral ACC, comprising subgenual and pregenual parts, has been ascribed to the autonomic component of sexual behavior mediating physiological readiness (Redoute et al, 2000). It is of note though that sgACC and pgACC have also been characterized as brain regions coding emotional contents in a wider context (Beckmann et al, 2009), of which autonomic reactions and self-referential processes may represent certain subsets. 
This suggests that paroxetine may lead to an impaired responsiveness of brain regions previously ascribed to the processing of emotional and autonomic functions that are associated with but also reach beyond the pure autonomic triggering of sexual arousal. Our observation that activation of the hypothalamus as a key autonomic component of sexual arousal (Arnow et al, 2002; Ferretti et al, 2005) did not show an effect of medication at the predefined statistical thresholds might be in line with this claim, although interpretation of this null result should be treated with caution.

A rather more specific role in the mediation of sexual dysfunction can be attributed to the MCC. In a PET study using erotic stimulation, activation of this brain region was found to correlate with penile tumescence (Redoute et al, 2000), and a recent study using fMRI could replicate this finding by demonstrating that the activation of the MCC correlated with penile tumescence in the early phase of sexual arousal and preceded physiological reactions (Moulier et al, 2006). Georgiadis et al (2010) reported blood flow changes in MCC and lateral hypothalamus that correlated with penis stimulation and perceived sexual arousal. In a recent study comparing effects of mirtazapine $v s$ SSRIs (paroxetine and fluoxetine) during fMRI of dynamic erotic stimulation, patients with major depression showed decreased activation under SSRIs (Kim et al, 2009) in cingulate areas in close proximity to the junction of the pMCC and posterior cingulate cortex. This pattern of results together with the present observation of decreased pMCC activation under paroxetine correlating with increased subjective sexual dysfunction supports a rather specific role of the MCC in mediating sexual functioning that is affected by SSRIs.

\section{SSRI-Related Alteration of Midbrain and Ventral Striatum Activation}

SSRI-dependent modulations of activity in midbrain and ventral striatum align with recent evidence indicating that the functioning of these brain regions is tuned by serotonin (Kranz et al, 2010). The positive activation of these regions upon erotic stimulation in the sense of a primary reward under placebo adds to the accepted interpretation of these brain regions as part of the dopaminergic reward system (Kranz et al, 2010). Despite the inconsistencies concerning the general role of serotonin in reward processing with reports on reward-increasing and reward-decreasing effects of serotonin, the latter results seem more prevalent. Particularly, diminished sexual desire and pleasure under SSRIs have been associated with the inhibition of dopaminergic reward circuits (Kranz et al, 2010). This inhibition of dopamine transmission by SSRIs has been shown to be mediated by 5-HT2 receptors (Alex and Pehek, 2007), and research in animals demonstrated inhibitory effects of serotonin in the lateral hypothalamus upon sexual behavior (Lorrain et al, 1997) and on dopamine release in the ventral striatum (Lorrain et al, 1999). A 5-HT2A receptor polymorphism in humans has been reported to modulate sexual arousal under SSRIs in patients with depression (Bishop et al, 2006). SSRIs have also been reported to be less effective in reducing reward-related symptoms of depression like loss of interest and motivation (Price et al, 2009).
In contrast, greater positive effects in this respect have been ascribed to antidepressants with enhancing effects on catecholamine levels (Nutt et al, 2007) like bupropion. Accordingly, paroxetine attenuated activation in brain regions that are associated with the rewarding, motivational aspects of sexual behavior.

\section{Activity Increases of Subcortical Regions Under Bupropion}

Consistent with the proposed opponency of dopamine and serotonin (Daw et al, 2002), effects under bupropion as a dopamine and norepinephrine reuptake inhibitor were almost contrary to the SSRI paroxetine in midbrain and ventral striatal areas. Furthermore, differential effects under bupropion were found over a prolonged time course during video stimulation even when compared with placebo. Modulation of ventral striatal activation by the subjectively perceived sexual intensity of a stimulus has been described before (Walter et al, 2008a), and changes in activity of the ventral striatum have been reported in various contexts of a rewarding sexual stimulation (Hamann et al, 2004; Stark et al, 2005). Dopamine agonists have been shown to induce penile erection in animals and humans (Carson, 2007) and have been suggested as promising agents for the treatment of sexual dysfunction (Albersen et al, 2010). Dopamine agonists have further been related to hypersexuality in the treatment of Parkinson's disease (for review, see Voon et al, 2009). Impaired recruitment of the midbrain was suggested as a correlate of decreased sexual interest in an fMRI study on pedophilia (Walter et al, 2007). These findings support the interpretation that increased activation of the ventral striatum and the midbrain as dopaminergic brain regions may represent a neural correlate of increased responsiveness to sexual stimuli triggered by the dopamine agonistic properties of bupropion. This effect may also explain the concomitantly increased activation of the thalamus, parahippocampus, and SLEA, as these structures are heavily interconnected. According to animal studies, the mediodorsal thalamus has subcortical connections to the ventral tegmental area and the midbrain's periaqueductal gray to the amygdala, the SLEA, and the hypothalamus (Erickson et al, 2004; Ongur and Price, 2000). The thalamus and amygdala have already been related to the emotional dimension of sexual arousal (Ferretti et al, 2005; Redoute et $a l, 2000$ ), and particularly the mediodorsal proportion of the thalamus was found active for both erotic and emotional stimulation (Walter et al, 2008b). Recently, a region around the SLEA with similar stereotactic coordinates as in the present study was reported to show the strongest blood flow changes when penile erection and perceived sexual arousal peaked and was suggested to potentially relate to orgasmic pleasure (Georgiadis et al, 2010). Together with cortical regions like the MCC, posterior cingulate, and anterior insula, the network of these subcortical regions has been referred to as the 'salience network', integrating functions of conflict monitoring, autonomic functions, and reward processing (Seeley et al, 2007). Activation in pMCC was increased under bupropion as the cortical part of the network that has been suggested to represent the approach component of sexual arousal (Redoute et al, 2000). 


\section{Unidirectional Effects of Both Antidepressants}

Despite opposite effects in extended networks, both drugs led to a decrease of activation compared with placebo in one subregion of the cingulate cortex, the aMCC. As both drugs otherwise led to differential effects upon processing erotic stimulation, this unidirectional effect does not integrate in a stimulation-bound interpretation. Alternatively, one might assume that the activation decrease in the aMCC may reflect an alteration of neural functioning not necessarily interfering with sexual functioning. This might be supported by the observation that both drugs also led to unidirectional behavioral effects with impaired performance on the divided attention task. This task puts the subject in a dual-task situation where task-set processes have been suggested to constitute a core resource of limited capacity shared across different tasks (Dosenbach et al, 2006). Midcingulate activation in the vicinity of the aMCC focus was suggested to be part of a frontoparietal network, exerting active and adaptive online control that helps to direct selective attention (Dosenbach et al, 2007). Although we could not observe substantial across-subject correlations between reaction time and decreased activity of the aMCC, one might speculate that impaired divided attention and decreased aMCC activation may both result from an impaired task-set processing under both drugs presumably via different receptor pathways. Interactions of dopamine receptors with NMDA receptors (Chen and Yang, 2002) with their particularly high density in aMCC (but not other ACC subregions; Palomero-Gallagher et al, 2009) could help to explain the differential effects of bupropion on aMCC and pMCC.

\section{CONCLUSION}

Enhancement of serotonergic and noradrenergic/dopaminergic neurotransmission led to differential effects in the subjective experiences of sexual functioning accompanied by opposite drug effects on local neural activities upon erotic stimulation. Overall, most brain activations elicited by erotic movies were reduced under paroxetine but not under bupropion. Particularly, under paroxetine, neural activities decreased in the ventral striatum, midbrain, and distinct areas along the cingulate gyrus. These regions have already been described by others to mediate aspects of motivational and emotional processes related to sexual behavior, although their specific role awaits further research. The combined noradrenalin/dopamine reuptake inhibitor bupropion showed opposite fMRI effects and was associated with a prolonged and increased activation of brain regions previously related to the processing of rewarding and salient stimuli. If clinically possible, this suggests enhancement of dopaminergic transmission as a plausible add-on treatment to counteract the side effects of antidepressant SSRI medication on sexual functioning that impose a high risk on medication adherence and hence therapeutic success.

\section{ACKNOWLEDGEMENTS}

We thank Professor Dr C Hiemke and his staff at the University of Mainz (Germany), Department of Psychiatry and Psychotherapy, for measurements of paroxetine and bupropion blood serum levels.

\section{DISCLOSURE}

The authors declare no conflict of interest.

\section{REFERENCES}

Albersen M, Shindel AW, Mwamukonda KB, Lue TF (2010). The future is today: emerging drugs for the treatment of erectile dysfunction. Expert Opin Emerg Drugs 15: 467-480.

Alex KD, Pehek EA (2007). Pharmacologic mechanisms of serotonergic regulation of dopamine neurotransmission. Pharmacol Ther 113: 296-320.

Arce E, Simmons AN, Lovero KL, Stein MB, Paulus MP (2008). Escitalopram effects on insula and amygdala BOLD activation during emotional processing. Psychopharmacology (Berl) 196: 661-672.

Arnow BA, Desmond JE, Banner LL, Glover GH, Solomon A, Polan ML et al (2002). Brain activation and sexual arousal in healthy, heterosexual males. Brain 125: 1014-1023.

Baldwin D, Mayers A (2003). Sexual side-effects of antidepressant and antipsychotic drugs. Adv Psychiatr Treat 9: 202-210.

Bauer M, Monz BU, Montejo AL, Quail D, Dantchev N, Demyttenaere $\mathrm{K}$ et al (2008). Prescribing patterns of antidepressants in Europe: results from the factors influencing depression endpoints research (FINDER) study. Eur Psychiatry 23: 66-73.

Beckmann M, Johansen-Berg H, Rushworth MF (2009). Connectivity-based parcellation of human cingulate cortex and its relation to functional specialization. J Neurosci 29: 1175-1190.

Bishop JR, Moline J, Ellingrod VL, Schultz SK, Clayton AH (2006). Serotonin 2A -1438 G/A and G-protein Beta3 subunit C825T polymorphisms in patients with depression and SSRI-associated sexual side-effects. Neuropsychopharmacology 31: 2281-2288.

Blier P, Chaput Y, de Montigny C (1988). Long-term 5-HT reuptake blockade, but not monoamine oxidase inhibition, decreases the function of terminal 5-HT autoreceptors: an electrophysiological study in the rat brain. Naunyn Schmiedebergs Arch Pharmacol 337: 246-254.

Bull SA, Hunkeler EM, Lee JY, Rowland CR, Williamson TE, Schwab JR et al (2002). Discontinuing or switching selective serotonin-reuptake inhibitors. Ann Pharmacother 36: 578-584.

Carson III CC (2007). Central nervous system-acting agents and the treatment of erectile and sexual dysfunction. Curr Urol Rep 8: $472-476$.

Chen L, Yang CR (2002). Interaction of dopamine D1 and NMDA receptors mediates acute clozapine potentiation of glutamate EPSPs in rat prefrontal cortex. J Neurophysiol 87: 2324-2336.

Clayton AH, Pradko JF, Croft HA, Montano CB, Leadbetter RA, Bolden-Watson C et al (2002). Prevalence of sexual dysfunction among newer antidepressants. J Clin Psychiatry 63: 357-366.

Clayton AH, Zajecka J, Ferguson JM, Filipiak-Reisner JK, Brown MT, Schwartz GE (2003). Lack of sexual dysfunction with the selective noradrenaline reuptake inhibitor reboxetine during treatment for major depressive disorder. Int Clin Psychopharmacol 18: 151-156.

Coleman CC, King BR, Bolden-Watson C, Book MJ, Segraves RT, Richard N et al (2001). A placebo-controlled comparison of the effects on sexual functioning of bupropion sustained release and fluoxetine. Clin Ther 23: 1040-1058.

Daw ND, Kakade S, Dayan P (2002). Opponent interactions between serotonin and dopamine. Neural Netw 15: 603-616.

Dosenbach NUF, Fair DA, Miezin FM, Cohen AL, Wenger KK, Dosenbach RAT et al (2007). Distinct brain networks for 
adaptive and stable task control in humans. Proc Natl Acad Sci USA 104: 11073-11078.

Dosenbach NUF, Visscher KM, Palmer ED, Miezin FM, Wenger KK, Kang HC et al (2006). A core system for the implementation of task sets. Neuron 50: 799-812.

Dunn JA, Arakawa R, Greist JH, Clayton AH (2007). Assessing the onset of antidepressant-induced sexual dysfunction using interactive voice response technology. J Clin Psychiatry 68: 525-532.

Erickson SL, Melchitzky DS, Lewis DA (2004). Subcortical afferents to the lateral mediodorsal thalamus in cynomolgus monkeys. Neuroscience 129: 675-690.

Ferretti A, Caulo M, Del Gratta C, Di Matteo R, Merla A, Montorsi $\mathrm{F}$ et al (2005). Dynamics of male sexual arousal: distinct components of brain activation revealed by fMRI. Neuroimage 26: 1086-1096.

Gaebel W, Falkai P (2001). Praxisleitlinien in Psychiatrie und Psychotherapie Bd. 5: Behandlungsleitlinie Affektive Erkrankungen. 1st edn. Steinkopff-Verlag: Darmstadt.

Georgiadis JR, Farrell MJ, Boessen R, Denton DA, Gavrilescu M, Kortekaas R et al (2010). Dynamic subcortical blood flow during male sexual activity with ecological validity: a perfusion fMRI study. Neuroimage 50: 208-216.

Giuliano F, Clement P (2006). Serotonin and premature ejaculation: from physiology to patient management. Eur Urol 50: 454-466.

Gregorian RS, Golden KA, Bahce A, Goodman C, Kwong WJ, Khan ZM (2002). Antidepressant-induced sexual dysfunction. Ann Pharmacother 36: 1577-1589.

Haddjeri N, Blier P, de Montigny C (1998). Long-term antidepressant treatments result in a tonic activation of forebrain 5-HT1A receptors. J Neurosci 18: 10150-10156.

Hamann S, Herman RA, Nolan CL, Wallen K (2004). Men and women differ in amygdala response to visual sexual stimuli. Nat Neurosci 7: 411-416.

Hautzinger M, Bailer M (1993). Allgemeine Depressionsskala (ADS). Beltz: Weinheim.

Kim W, Jin BR, Yang WS, Lee KU, Juh RH, Ahn KJ et al (2009). Treatment with selective serotonin reuptake inhibitors and mirtazapine results in differential brain activation by visual erotic stimuli in patients with major depressive disorder. Psychiatry Invest 6: 85-95.

Kranz GS, Kasper S, Lanzenberger R (2010). Reward and the serotonergic system. Neuroscience 166: 1023-1035.

Labbate LA, Lare SB (2001). Sexual dysfunction in male psychiatric outpatients: validity of the Massachusetts General Hospital Sexual Functioning Questionnaire. Psychother Psychosom 70: 221-225.

Lingjaerde O, Ahlfors UG, Bech P, Dencker SJ, Elgen K (1987). The UKU side effect rating scale. A new comprehensive rating scale for psychotropic drugs and a cross-sectional study of side effects in neuroleptic-treated patients. Acta Psychiatr Scand Suppl 334: $1-100$.

Lorrain DS, Matuszewich L, Friedman RD, Hull EM (1997). Extracellular serotonin in the lateral hypothalamic area is increased during the postejaculatory interval and impairs copulation in male rats. J Neurosci 17: 9361-9366.

Lorrain DS, Riolo JV, Matuszewich L, Hull EM (1999). Lateral hypothalamic serotonin inhibits nucleus accumbens dopamine: implications for sexual satiety. J Neurosci 19: 7648-7652.

Mayberg HS, Lozano AM, Voon V, McNeely HE, Seminowicz D, Hamani C et al (2005). Deep brain stimulation for treatmentresistant depression. Neuron 45: 651-660.

McCabe C, Mishor Z, Cowen PJ, Harmer CJ (2010). Diminished neural processing of aversive and rewarding stimuli during selective serotonin reuptake inhibitor treatment. Biol Psychiatry 67: 439-445.

Modell JG, May RS, Katholi CR (2000). Effect of bupropion-SR on orgasmic dysfunction in nondepressed subjects: a pilot study. J Sex Marital Ther 26: 231-240.
Montejo AL, Llorca G, Izquierdo JA, Rico-Villademoros F (2001). Incidence of sexual dysfunction associated with antidepressant agents: a prospective multicenter study of 1022 outpatients. Spanish Working Group for the Study of Psychotropic-Related Sexual Dysfunction. J Clin Psychiatry 62(Suppl 3): 10-21.

Moulier V, Mouras H, Pelegrini-Issac M, Glutron D, Rouxel R, Grandjean B et al (2006). Neuroanatomical correlates of penile erection evoked by photographic stimuli in human males. Neuroimage 33: 689-699.

Mouras H (2004). Identifying distinct components in the cerebral treatment of visual sexual information through functional neuroimaging. J Soc Biol 198: 247-253.

Nutt D, Demyttenaere K, Janka Z, Aarre T, Bourin M, Canonico PL et al (2007). The other face of depression, reduced positive affect: the role of catecholamines in causation and cure. J Psychopharmacol 21: 461-471.

Ongur D, Price JL (2000). The organization of networks within the orbital and medial prefrontal cortex of rats, monkeys and humans. Cereb Cortex 10: 206-219.

Palomero-Gallagher N, Vogt BA, Schleicher A, Mayberg HS, Zilles K (2009). Receptor architecture of human cingulate cortex: evaluation of the four-region neurobiological model. Hum Brain Mapp 30: 2336-2355.

Patel K, Hellstrom WJ (2009). Central regulation of ejaculation and the therapeutic role of serotonergic agents in premature ejaculation. Curr Opin Investig Drugs 10: 681-690.

Pessiglione M, Seymour B, Flandin G, Dolan RJ, Frith CD (2006). Dopamine-dependent prediction errors underpin reward-seeking behaviour in humans. Nature 442: 1042-1045.

Phan KL, Wager T, Taylor SF, Liberzon I (2002). Functional neuroanatomy of emotion: a meta-analysis of emotion activation studies in PET and fMRI. Neuroimage 16: 331-348.

Price J, Cole V, Goodwin GM (2009). Emotional side-effects of selective serotonin reuptake inhibitors: qualitative study. $\mathrm{Br} \mathrm{J}$ Psychiatry 195: 211-217.

Radloff LS (1977). The CES-D scale: a self-report depression scale for research in the general population. Appl Psychol Measurement 1: 385-401.

Redoute J, Stoleru S, Gregoire MC, Costes N, Cinotti L, Lavenne F et al (2000). Brain processing of visual sexual stimuli in human males. Hum Brain Mapp 11: 162-177.

Reinecke A, Schöps D, Hoyer J (2006). Sexuelle Dysfunktionen bei Patienten einer verhaltenstherapeutischen Hochschulambulanz: Häufigkeit, Erkennen, Behandlung. Verhaltenstherapie 16: 166-172.

Ressler KJ, Mayberg HS (2007). Targeting abnormal neural circuits in mood and anxiety disorders: from the laboratory to the clinic. Nat Neurosci 10: 1116-1124.

Seeley WW, Menon V, Schatzberg AF, Keller J, Glover GH, Kenna $\mathrm{H}$ et al (2007). Dissociable intrinsic connectivity networks for salience processing and executive control. I Neurosci 27: 2349-2356.

Serretti A, Chiesa A (2009). Treatment-emergent sexual dysfunction related to antidepressants: a meta-analysis. J Clin Psychopharmacol 29: 259-266.

Simmons AN, Arce E, Lovero KL, Stein MB, Paulus MP (2009). Subchronic SSRI administration reduces insula response during affective anticipation in healthy volunteers. Int J Neuropsychopharmacol 12: 1009-1020.

Stark R, Schienle A, Girod C, Walter B, Kirsch P, Blecker C et al (2005). Erotic and disgust-inducing pictures-differences in the hemodynamic responses of the brain. Biol Psychol 70: 19-29.

Vogt BA, Berger GR, Derbyshire SW (2003). Structural and functional dichotomy of human midcingulate cortex. Eur $J$ Neurosci 18: 3134-3144.

Voon V, Fernagut PO, Wickens J, Baunez C, Rodriguez M, Pavon N et al (2009). Chronic dopaminergic stimulation in Parkinson's 
disease: from dyskinesias to impulse control disorders. Lancet Neurol 8: 1140-1149.

Waldinger MD, Berendsen HH, Blok BF, Olivier B, Holstege G (1998). Premature ejaculation and serotonergic antidepressantsinduced delayed ejaculation: the involvement of the serotonergic system. Behav Brain Res 92: 111-118.

Walter M, Bermpohl F, Mouras H, Schiltz K, Tempelmann C, Rotte $\mathrm{M}$ et al (2008a). Distinguishing specific sexual and general emotional effects in fMRI-subcortical and cortical arousal during erotic picture viewing. Neuroimage 40: 1482-1494.

Walter M, Stadler J, Tempelmann C, Speck O, Northoff G (2008b). High resolution $\mathrm{fMRI}$ of subcortical regions during visual erotic stimulation at 7 T. Magma 21: 103-111.

Walter M, Witzel J, Wiebking C, Gubka U, Rotte M, Schiltz K et al (2007). Pedophilia is linked to reduced activation in hypothalamus and lateral prefrontal cortex during visual erotic stimulation. Biol Psychiatry 62: 698-701.

Supplementary Information accompanies the paper on the Neuropsychopharmacology website (http://www.nature.com/npp) 